\title{
Adiposity, fat depots and the prediction of stroke
}

\author{
Maciej Haberka ${ }^{1}$, Andrzej Kubicius ${ }^{2}$, Monika Starzak ${ }^{3}$, \\ Małgorzata Partyka ${ }^{4}$, Zbigniew Gąsior ${ }^{1}$ \\ ${ }^{1}$ Department of Cardiology, SHS, Medical University of Silesia, Katowice, Poland \\ ${ }^{2}$ Department of Cardiology, Upper Silesia Medical Center, Katowice, Poland \\ ${ }^{3}$ Department of Cardiology, General Hospital No. 4, Gliwice, Poland \\ ${ }^{4}$ Department of Diagnostic Imaging, City Hospital, Chorzow, Poland
}

\begin{abstract}
Background: Despite the progress in research, the utility of clinical assessment for the prediction of stroke is limited. The aim herein, was to evaluate the predictive values of major ultrasound indexes of carotid artery and fat depots for stroke in patients with high and very high cardiovascular (CV) risk.

Methods: The study group included 364 patients (age: $61.3 \pm 7.2$ years old) with typical CV risk factors scheduled for elective coronary angiography (2012-2013). A comprehensive baseline assessment included the following ultrasound indexes: carotid artery intima-media thickness (IMT), extra-media thickness (EMT), epicardial (EFT) and pericardial fat thickness (PFT), abdominal subcutaneous $(A S F)$ and visceral fat $(A V F)$ and combined periarterial adipose tissue intima-media adventitia (PATIMA) index. Afterwards, all patients were followed for $80.9 \pm 7.1$ months.

Results: There were 23 strokes and 25 cases with new-onset atrial fibrillation during follow-up. Receiver operating characteristics (ROC) analysis showed, that selected clinical parameters (age, waist circumference [WC], waist-hip ratio [WHR]) and ultrasound indexes (EFT: area under curve [AUC] $0.672, p<0.01$ and PATIMA index: AUC 0.658, $p<0.01)$ were predictive for stroke. However, their predictive values showed no significant differences $(p=N S)$. The baseline body mass index (BMI) was the only parameter, which showed a prediction for new-onset atrial fibrillation (BMI $>33 \mathrm{~kg} / \mathrm{m}^{2}$ : sensitivity 65\%, specificity 76\%).

Conclusions: It was found that age, WC and echocardiographic EFT revealed significant predictive values for stroke. Both WC and EFT showed a very high NPV suggesting that they should be implemented into the clinical practice as a tool affirming a very low risk of stroke. (Cardiol J)
\end{abstract}

Key words: epicardial fat, extra-media thickness, intima-media thickness, atrial fibrillation, stroke

\section{Introduction}

Cerebrovascular disease is an important cause of disability and mortality in developed countries [1]. Atrial fibrillation (AF) is one of the major causes of ischemic stroke [2] and cardioembolism is the most frequent cause of cryptogenic events [3]. Obesity is another world-wide medical problem increasing the risk of several cardiovascular $(\mathrm{CV})$ diseases and complications [4]. However, the estimation of $\mathrm{CV}$ risk in patients with several comorbidities is difficult. Obesity has a few phenotypes, which are associated with different risk, especially in patients with chronic CV diseases [5]. This is explained by fat depots with a more or less active role in metabolism or local pathophysiology of arterial wall or heart muscle [6]. Previous studies showed that perivascular fat thickness is associ-

Address for correspondence: Maciej Haberka, MD, PhD, Department of Cardiology, SHS, Medical University of Silesia, ul. Ziołowa 45/47, 40-635 Katowice, Poland, tel: +48 32 2527407, fax: + 4832 2523032, e-mail: mhaberka@op.pl

Received: 17.01.2021 Accepted: 2.07.2021 Early publication date: 25.10.2021

This article is available in open access under Creative Common Attribution-Non-Commercial-No Derivatives 4.0 International (CC BY-NC-ND 4.0) license, allowing to download articles and share them with others as long as they credit the authors and the publisher, but without permission to change them in any way or use them commercially. 
ated with cardiometabolic risk [7-9], peripheral atherosclerosis [10] and the severity of coronary artery disease (CAD) [11, 12]. Whereas abdominal visceral adipose tissue is more associated with metabolic risk $[9,10]$.

Despite the great progress in research, the utility of clinical assessment for the prediction of stroke is still limited. Therefore, the aim herein, was to evaluate the predictive values of major ultrasound indexes of carotid artery and fat depots for stroke in patients with high and very high CV risk.

\section{Methods}

\section{Study group}

All the consecutive patients (age: $50-75$ years old) scheduled for CV diagnostics in the Department of Cardiology (2012-2013) were recruited to the study group. The following exclusion criteria were used at the screening: heart failure, significant heart valve defects, chronic inflammatory diseases, neoplastic diseases in the prior 5 years, secondary causes of obesity or prior interventions in obesity, a $10 \%$ unintentional weight loss, a poor carotid artery image quality and a genetic predisposition for CV diseases.

Subjects completed the study at the Department of Cardiology at the Medical University of Silesia (SUM). The protocol was approved by the SUM Ethics Committee. This work was supported by a research non-commercial grant from the Medical University of Silesia (KNW-1-029/N/8/K).

\section{Clinical characteristics}

A comprehensive clinical assessment and the following ultrasound indexes were obtained in all patients: abdominal subcutaneous fat (ASF), abdominal visceral fat (AVF), carotid intima-media thickness (IMT), carotid extra-media thickness (EMT), epicardial fat thickness (EFT), pericardial fat thickness (PFT) and the periarterial adipose tissue intima-media adventitia (PATIMA) index.

Hyperlipidemia was determined based on plasma lipid levels or prior diagnosis and current treatment [13]. The diagnosis of hypertension was confirmed by the office blood pressure or prior diagnosis and current treatment [13]. Diabetes mellitus (DM) was reported in patients with prior diagnosis or abnormal fasting plasma glucose concentration $(\geq 126 \mathrm{mg} / \mathrm{dL})$ or $\mathrm{HbA1c}(\geq 6.5 \%)$ or 2 -h post-load plasma glucose $(\geq 200 \mathrm{mg} / \mathrm{dL})$ in case of discrepancies $[14,15]$.

Obesity was classified according to body mass index $\left(\mathrm{BMI}=\right.$ body mass $[\mathrm{kg}] /$ height $\left.[\mathrm{m}]^{2}\right)$ as normal weight (18.5-24.9 kg/m²), overweight $\left(25.0-29.9 \mathrm{~kg} / \mathrm{m}^{2}\right)$ and obesity $\left(\geq 30.0 \mathrm{~kg} / \mathrm{m}^{2}\right)$ : class $1\left(30.0-34.9 \mathrm{~kg} / \mathrm{m}^{2}\right)$, class $2\left(35.0-39.9 \mathrm{~kg} / \mathrm{m}^{2}\right)$ and class $3\left(\geq 40.0 \mathrm{~kg} / \mathrm{m}^{2}\right)$. Chronic kidney disease was determined based on estimated glomerular filtration rate $\left(<60 \mathrm{~mL} / \mathrm{min} / 1.73 \mathrm{~m}^{2}\right)$ or prior diagnosis and treatment. Waist circumference (WC: midpoint between the lowest rib and the iliac crest) and hip circumference $(\mathrm{HC})$ were measured with a tape at the end of expiration. Coronary artery disease was defined as stenosis $\geq 50 \%$ in any major coronary artery assessed in coronary angiography. Finally, CV risk was estimated for each of the study patients based on the European Society of Cardiology guidelines [13].

\section{Ultrasound indexes}

All the ultrasound scans were analyzed offline blinded to the patient's data with the intra- and interobserver variability as shown previously [11].

\section{Carotid artery indexes: IMT and EMT}

All the patients were in a supine position at the ECG-gated ultrasound examination of both carotid arteries (GE Vivid 9, Milwaukee, US linear transducer 9-12 MHz). All the images were recorded by an experienced researcher using constant settings and once the recruitment was finished, images with the region of interest were analyzed offline blinded to patient's data. Common carotid artery IMT was measured according to the Mannheim Consensus Guidelines on a $10 \mathrm{~mm}$ -length segment starting $5 \mathrm{~mm}$ proximally to the carotid bulb using a semi-automated GE software [16]. Carotid IMT was an average value of both sides' carotid arteries.

Carotid EMT was measured in the images obtained in an alternative (to IMT) scanning of the carotid vessels and the method was described in our previous studies [11]. In brief, EMT is the distance between the carotid media-adventitia border and the jugular wall-lumen interface averaged from both common carotid arteries (CCA) with visualization of the zoomed interface between the near wall of the distal segment of the CCA and the neighboring jugular vein. It is measured manually in a standardized protocol including a $7 \mathrm{~mm}$ segment starting $3 \mathrm{~mm}$ proximal to the bulb. The mean EMT values were averaged from the serial measurements taken at end-diastole ( 5 consecutive beats) in both sides' carotid arteries.

\section{Cardiac fat: EFT and PFT}

Epicardial adipose tissue (EFT) was evaluated using transthoracic echocardiography according to 
the most frequent and accepted method [17, 18]. Two-dimensional long and short axis parasternal views were obtained and recorded using GE Vivid 9 ultrasound machine with a $1.5-4.5 \mathrm{MHz}$ transducer. The EFT was identified as an area between epicardium and visceral layer of the pericardium. The PFT was determined as an area between the visceral layer and parietal pericardium layer. Both (EFT and PFT) were measured perpendicularly to the right ventricle free wall at the level of the aortic annulus. The maximum values were measured both at the end-diastole during 5 consecutive beats and a mean value was obtained.

\section{Abdominal fat: ASF and AVF}

The transducer $(7.5 \mathrm{MHz}$ for ASF or $3.5 \mathrm{MHz}$ for AVF) was placed on the skin ( $1 \mathrm{~cm}$ above umbilicus; transverse view) as lightly as possible to prevent compression of the layers and to perform a breath-hold measurements. The ASF was measured as the distance between the skin and external surface of the rectus abdominis muscle. The AVF was determined as the space between the internal surface of the rectus abdominis muscle and anterior wall of the aorta [7].

\section{Ultrasound combined indexes}

All the ultrasound indexes (carotid IMT and EMT, cardiac EFT) were used in analysis as separate mean values. Each of them represents various tissue components and may correspond to different aspects of cardiovascular risk. Therefore, combined indexes (PATIMA) were used. The PATIMA index was developed recently [11] and it was calculated according to the following formula: PATIMA $[\mathrm{u}]=$ $(\mathrm{EMT} / \mathrm{BMI} \times 35)+\mathrm{IMT}+(\mathrm{EFT} \times 60)$.

\section{Clinical follow-up}

All the clinical characteristics and ultrasound indexes were obtained prospectively at the time of enrollment (2012-2013). Afterwards, the Regional Branch of the National Health System (NHS) provided medical data regarding all the study patients. Data included inpatients and outpatients with the main diagnosis, concomitant diseases and treatment in the time period up to the end of 2019. A stroke was determined if it was either the main or concomitant diagnosis or a cause of death reported by the hospital or outpatient. A new-onset $\mathrm{AF}$ (NOAF) was determined if $\mathrm{AF}$ was reported in the follow-up time and was not present at the time of the study enrollment (2012-2013). Finally, NHS medical data regarding mortality was also collected.

\section{Statistical analysis}

All results presented in the text, tables and figure are expressed as means \pm standard deviation or number and percentage. The results' normal distribution was analyzed with the Kolmogorov-Smirnov test. Associations between parameters were assessed using the Pearson correlation analysis. To determine the best cut-off of particular clinical parameters or ultrasound indexes, the receiver operating characteristic (ROC) curves were used providing sensitivity, specificity, positive (PPV) and negative (NPV) predictive values for a stroke and a NOAF. Moreover, multivariable logistic regression models were employed to identify variables independently associated with follow-up events. A value $\mathrm{p}<0.05$ was considered statistically significant. Statistical analysis was undertaken using MedCalc (version 18.5, MedCalc Software).

\section{Results}

\section{Study group characteristics}

The study group included 364 patients (age: $61.3 \pm 7.2$ years old) with high or very high CV risk. All the patients used a standard pharmacotherapy, including beta-blockers (95\%), acetylsalicylic acid (95\%), lipid lowering treatment (100\%) - statins (95\%), fibrates (3\%), ezetimibe $(2 \%)$, angiotensin-converting enzyme inhibitors or angiotensin receptor blockers (86\%), diuretics (30\%), calcium channel blockers (26\%) and nitrates (18\%).

The study patients revealed several $\mathrm{CV}$ risk factors as follows: dyslipidemia (100\%), hypertension $(89 \%)$, obesity $(49 \%)$, diabetes $(35 \%)$, and smoking (29\%). Most patients had CAD (70\%), one fifth $(22 \%)$ had peripheral artery disease and carotid artery stenosis $(\geq 50 \%)$ was found in 51 patients. Furthermore, 42 patients were found to have $\mathrm{AF}$ at baseline and medical history revealed prior myocardial infarction in $21 \%$ and stroke in $6 \%$ of the cases (Table 1 ).

The mean measures of ultrasound indexes are presented in Table 2 .

The baseline carotid IMT showed a weak association with carotid EMT $(r=0.25, \mathrm{p}<0.0001)$, $\operatorname{PFT}(\mathrm{r}=0.1, \mathrm{p}=0.01)$ and AVF $(\mathrm{r}=0.1, \mathrm{p}<0.01)$. None of the other ultrasound indexes showed a significant association. Moreover, EFT showed a weak association with BMI $(\mathrm{r}=0.2 ; \mathrm{p}<0.01)$ and no correlation with waist-hip ratio (WHR; $\mathrm{p}=0.8)$ or $\mathrm{WC}(\mathrm{p}=0.2)$. The PATIMA combined index also showed a weak association with BMI $(\mathrm{r}=0.2 ; \mathrm{p}=0.001)$ and no association with WHR $(\mathrm{p}=0.5)$ or $\mathrm{WC}(\mathrm{p}=0.8)$. 
Table 1. Clinical characteristics of the study group.

\begin{tabular}{lc}
\hline & $\begin{array}{c}\text { Mean } \pm \text { SD } \\
\text { or no. (\%) }\end{array}$ \\
\hline Age [years] & $61.3 \pm 7.2$ \\
Female/male & $131(36 \%) / 233(64 \%)$ \\
Diabetes & $130(36 \%)$ \\
Lipid-lowering treatment & $364(100 \%)$ \\
Total cholesterol [mg/dL] & $175 \pm 45$ \\
LDL-C [mg/dL] & $102 \pm 41$ \\
HDL-C [mg/dL] & $44 \pm 12$ \\
Triglycerides [mg/dL] & $141 \pm 84$ \\
Hypertension & $325(89 \%)$ \\
Chronic kidney disease & $17(5 \%)$ \\
Coronary artery disease & $255(70 \%)$ \\
Prior myocardial infarction & $77(21 \%)$ \\
Peripheral artery disease & $82(22 \%)$ \\
Carotid artery stenosis $\geq 50 \%$ & $51(14 \%)$ \\
Prior stroke & $22(6 \%)$ \\
Smoker or ex-smoker & $258(71 \%)$ \\
Body mass index [kg/m $\left.{ }^{2}\right]$ & $30.8 \pm 6$ \\
Obesity & $182(50 \%)$ \\
Risk factors & $4.8 \pm 1.5$ \\
Very high/high CV risk & $302(83 \%) / 62(17 \%)$ \\
\hline
\end{tabular}

CV - cardiovascular; LDL-C - low density lipoprotein cholesterol; HDL-C - high density lipoprotein cholesterol; SD — standard deviation

Table 2. Ultrasound indexes of cardiovascular risk.

\begin{tabular}{lc}
\hline & $\begin{array}{c}\text { Mean } \pm \text { SD } \\
\text { or no. }(\%)\end{array}$ \\
\hline Abdominal visceral fat $[\mathrm{mm}]$ & $76 \pm 26$ \\
Abdominal subcutaneous fat $[\mathrm{mm}]$ & $27.5 \pm 11$ \\
Carotid intima-media thickness $[\mu \mathrm{m}]$ & $915 \pm 360$ \\
Carotid extra-media thickness $[\mu \mathrm{m}]$ & $793 \pm 124$ \\
Epicardial fat thickness [mm] & $3.51 \pm 1.53$ \\
Pericardial fat thickness [mm] & $9 \pm 6.3$ \\
PATIMA combined index [u] & $211 \pm 96$ \\
\hline
\end{tabular}

PATIMA - periarterial adipose tissue intima-media adventitia; $\mathrm{SD}$ - standard deviation

\section{Clinical prognosis and follow-up}

The baseline clinical characteristics and ultrasound indexes were used in the ROC analysis in prediction of stroke and NOAF. All the study patients were followed for $80.9 \pm 7.1$ months. There were 50 deaths in the primary study group during follow-up. There were 23 strokes in 23 patients

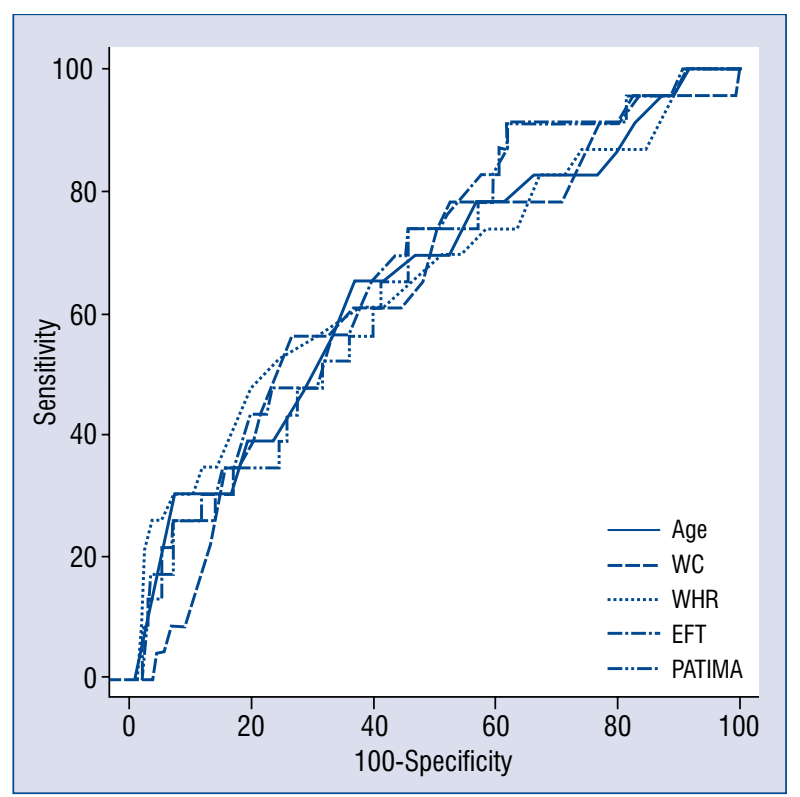

Figure 1. The receiver operating characteristics curves representing selected clinical parameters and ultrasound indexes with significant prediction of stroke; EFT - epicardial fat thickness; PATIMA — periarterial adipose tissue intima-media adventitia; WC - waist circumference; WHR - waist-hip ratio.

(4 patients died) and 25 cases with NOAF during the follow-up. The ROC analysis showed, that selected clinical parameters (age, WC, WHR) and ultrasound indexes (EFT and PATIMA combined index) were predictive for stroke. Their predictive values showed no significant differences $(p=N S)$. However, a multivariable logistic regression model including those five parameters showed that only EFT (odds ratio [OR] 1.4; 95\% confidence interval [CI] 0.5-2.3; $\mathrm{p}=0.04$ ) and PATIMA combined index (OR 2.1; 95\% CI 1.01-4.22; $\mathrm{p}=0.04$ ). Finally, baseline BMI was the only parameter, which showed a prediction for NOAF, which occurred during a follow-up (BMI $>33 \mathrm{~kg} / \mathrm{m}^{2}$ : sensitivity $65 \%$, specificity $76 \%$, PPV 14\%, NPV 97\%; Fig. 1 , Table 3).

\section{Discussion}

This was the first prospective study providing a complete analysis of clinical risk factors and major ultrasound indexes in the prediction for stroke among patients with at least high $\mathrm{CV}$ risk.

All the major ultrasound indexes were used, which are related to various tissue components, including subcutaneous or visceral adipose tissue, perivascular fat or various layers of the arterial 
Tables 3. The baseline characteristics and ultrasound indexes in prediction of clinical events in the receiver operating characteristics analysis.

\begin{tabular}{|c|c|c|c|c|}
\hline & \multicolumn{2}{|c|}{ Stroke } & \multicolumn{2}{|c|}{ Atrial fibrillation } \\
\hline & AUC & $\mathbf{P}$ & AUC & $\mathbf{P}$ \\
\hline Age & 0.661 & 0.002 & 0.557 & 0.32 \\
\hline Total number of $\mathrm{CV}$ risk factors & 0.535 & 0.57 & 0.5 & 0.98 \\
\hline LDL-C & 0.571 & 0.2 & 0.527 & 0.62 \\
\hline Triglycerides & 0.636 & 0.1 & 0.501 & 0.9 \\
\hline Body mass index & 0.542 & 0.45 & 0.694 & 0.001 \\
\hline Body fat & 0.517 & 0.8 & 0.555 & 0.38 \\
\hline Waist circumference & 0.640 & 0.01 & 0.602 & 0.09 \\
\hline Waist-hip ratio & 0.659 & 0.01 & 0.515 & 0.8 \\
\hline Abdominal visceral fat & 0.577 & 0.15 & 0.540 & 0.47 \\
\hline Abdominal subcutaneous fat & 0.526 & 0.6 & 0.6 & 0.09 \\
\hline Carotid intima-media thickness & 0.591 & 0.13 & 0.538 & 0.52 \\
\hline Carotid extra-media thickness & 0.540 & 0.5 & 0.503 & 0.95 \\
\hline Epicardial fat thickness & 0.672 & $<0.01$ & 0.556 & 0.45 \\
\hline Pericardial fat thickness & 0.513 & 0.8 & 0.520 & 0.7 \\
\hline PATIMA combined index & 0.658 & $<0.01$ & 0.563 & 0.39 \\
\hline
\end{tabular}

AUC - area under curve; CV — cardiovascular; LDL-C — low density lipoprotein cholesterol; PATIMA — periarterial adipose tissue intima-media adventitia

wall. The associations between baseline single ultrasound indexes were either statistically or clinically not significant, which suggests that they are related to different aspects of $\mathrm{CV}$ risk. The echocardiography EFT was the only single ultrasound index, which predicted strokes during a 7-year prospective follow-up. Although the combined PATIMA index was also found to have significant predictions for strokes, it is a derivative of the EFT measure and its predictive value was not superior to the EFT alone. Finally, the baseline BMI was the only parameter with a prediction for $\mathrm{NOAF}$ and ultrasound indexes failed to predict AF.

According to available research, the present study is the first to show that increased EFT $(>2.8 \mathrm{~mm})$ was predictive for stroke in a prospective follow-up. Three small and cross-sectional studies found a significantly higher EFT in patients with acute ischemic stroke compared to control groups [19-21]. Noteworthy, increased EFT was also found in young patients with embolic stroke of undetermined source [22]. Cho et al. [23] showed that patients with ischemic stroke and $\mathrm{AF}$ had higher EFT compared to subjects with stroke, but without AF. In the current study, EFT had a high sensitivity and a very high NPV suggesting that values lower than $2.8 \mathrm{~mm}$ indicate a very low risk of stroke. Given that epicardial fat is involved in various atherosclerotic diseases, increased EFT is not specific only for increased risk of stroke. Moreover, the present study showed that obesity has an important relation with $\mathrm{AF}$ and strokes. Although central obesity was found to be predictive for stroke, baseline BMI was the only parameter of adiposity with a predictive value for NOAF. All the ultrasound indexes failed to predict NOAF. The current study did not confirm the predictive value of EFT for AF, which was found in some other studies $[24,25]$.

A few major ultrasound indexes were used, which failed to predict NOAF or strokes. So far, carotid IMT was evidenced to have some predictive value for strokes $[26,27]$. The were no previous studies, which assessed other fat depots in relation to stroke. Despite the link between visceral fat and stroke, the present study did not confirm the relation between abdominal visceral adipse tissue (AVF) and the risk of stroke.

\section{Epicardial fat and stroke}

Epicardial adipocytes originate from brown adipose tissue and they have been shown to produce various types of cytokines [28, 29]. It has the same blood supply as the myocardium, with a close anatomic and functional relationship as well. It is a fat depot related not only to local ventricle mass 
or function, but it is also related to visceral adiposity and cardiometabolic risk [30, 31]. Epicardial fat is also involved in the pathogenesis of insulin resistance through endothelial function, insulin-mediated vasoreactivity, and muscle perfusion [32]. The EFT may be increased as a consequence of adipocytes' dysfunction and muscle proliferation [33]. Visceral obesity and an increasing number of adipocytes may induce a hypoxia and a pro-inflammatory state [33]. Moreover, increased release of pro-inflammatory cytokines induce lipolysis, oxidative stress and apoptosis within various depots of visceral adipose tissue [34]. Finally, excessive serum fatty acids link epicardial adipocytes, altered cardiac repolarization and arrhythmias [23]. Previous studies also suggest a link between EFT and the development of left atrial myopathy, increasing the risk of $\mathrm{AF}[35,36]$. Recent studies have shown that increased epicardial adiposity can directly modulate the electrophysiological properties of the heart and ion currents, causing higher arrhythmogenesis in left atrial myocytes, which contributes to an increased risk of AF [37]. There is also a well-established relation between general obesity, left atrial enlargement and changes in the histology of the atrial wall [38].

\section{Limitations of the study}

The present data regarding prospective clinical follow-up originated from the NHS which collects reports from all the medical centers. There was no prospective visit or active testing for unknown $\mathrm{AF}$ in the study. Therefore, some patients of the study could have undetected AF, which could have affected the present results. There is also a possibility that an undiagnosed stroke could have been a cause of death, which was not known, reported to NHS and was included in the analysis. However, this is also true for other similar studies.

\section{Conclusions}

This is the first study providing important results based on a comprehensive assessment of various ultrasound indexes reflecting vascular indexes or fat depots and a prospective follow-up. It was found that age, WC and echocardiographic EFT revealed significant predictive values for stroke. Both WC and EFT showed a very high NPV suggesting that they should be implemented into the clinical practice as a tool affirming a very low risk of stroke.

Conflict of interest: None declared

\section{References}

1. Towfighi A, Saver JL. Stroke declines from third to fourth leading cause of death in the United States: historical perspective and challenges ahead. Stroke. 2011; 42(8): 2351-2355, doi: 10.1161/ STROKEAHA.111.621904, indexed in Pubmed: 21778445.

2. Sanak D, Hutyra M, Kral M, et al. Paroxysmal atrial fibrillation in young cryptogenic ischemic stroke: a 3-week ECG Holter monitoring study. Biomed Pap Med Fac Univ Palacky Olomouc Czech Repub. 2015; 159(2): 283-287, doi: 10.5507/bp.2015.019, indexed in Pubmed: 25916280.

3. Mozaffarian D, Benjamin EJ, Go AS, et al. American Heart Association Statistics Committee and Stroke Statistics Subcommittee. Heart disease and stroke statistics--2015 update: a report from the American Heart Association. Circulation. 2015; 131: e29-e322.

4. Poirier P, Giles TD, Bray GA, et al. Obesity and cardiovascular disease: pathophysiology, evaluation, and effect of weight loss: an update of the 1997 American Heart Association Scientific Statement on Obesity and Heart Disease from the Obesity Committee of the Council on Nutrition, Physical Activity, and Metabolism. Circulation. 2006; 113(6): 898-918, doi: 10.1161/CIRCULATIONAHA.106.171016, indexed in Pubmed: 16380542.

5. Haberka M, Stolarz-Skrzypek K, Czarnecka D, et al. Overweight and grade I obesity in patients with cardiovascular disease: to treat or not to treat? Pol Arch Med Wewn. 2014; 124(12): 731-739, doi: 10.20452/pamw.2564, indexed in Pubmed: 25354500.

6. Haberka M, Machnik G, Kowalówka A, et al. Epicardial, paracardial, and perivascular fat quantity, gene expressions, and serum cytokines in patients with coronary artery disease and diabetes. Pol Arch Intern Med. 2019; 129(11): 738-746, doi: 10.20452/ pamw.14961, indexed in Pubmed: 31479091.

7. Haberka M, Gąsior Z. Carotid extra-media thickness in obesity and metabolic syndrome: a novel index of perivascular adipose tissue: extra-media thickness in obesity and metabolic syndrome. Atherosclerosis. 2015; 239(1): 169-177, doi: 10.1016/j. atherosclerosis.2014.12.058, indexed in Pubmed: 25617857.

8. Haberka M, Okopień B, Gąsior Z. Obesity, ultrasound indexes of fat depots and lipid goal attainment in patients with high and very high cardiovascular risk: a novel approach towards better risk reduction. Nutr Metab Cardiovasc Dis. 2016; 26(2): 123-133, doi: 10.1016/j.numecd.2015.10.012, indexed in Pubmed: 26830392.

9. Haberka M, Stolarz-Skrzypek K, Biedroń M, et al. Obesity, visceral fat, and hypertension-related complications. Metab Syndr Relat Disord. 2018; 16(10): 521-529, doi: 10.1089/met.2018.0062, indexed in Pubmed: 30183499.

10. Haberka M, Skilton M, Biedroń M, et al. Obesity, visceral adiposity and carotid atherosclerosis. J Diabetes Complications. 2019; 33(4): 302-306, doi: 10.1016/j.jdiacomp.2019.01.002, indexed in Pubmed: 30770289.

11. Haberka M, Gąsior Z. A carotid extra-media thickness, PATIMA combined index and coronary artery disease: Comparison with well-established indexes of carotid artery and fat depots. Atherosclerosis. 2015; 243(1): 307-313, doi: 10.1016/j.atherosclerosis.2015.09.022, indexed in Pubmed: 26414210.

12. Haberka M, Lelek M, Bochenek T, et al. Novel combined index of cardiometabolic risk related to periarterial fat improves the clinical prediction for coronary artery disease complexity. Atherosclerosis. 2018; 268: 76-83, doi: 10.1016/j.atherosclerosis.2017.09.015, indexed in Pubmed: 29195108.

13. Piepoli MF, Hoes AW, Agewall S, et al. 2016 European Guidelines on cardiovascular disease prevention in clinical practice: The 
Sixth Joint Task Force of the European Society of Cardiology and Other Societies on Cardiovascular Disease Prevention in Clinical Practice (constituted by representatives of 10 societies and by invited experts)Developed with the special contribution of the European Association for Cardiovascular Prevention \& Rehabilitation (EACPR). Eur Heart J. 2016; 37(29): 2315-2381, doi: 10.1093/eurheartj/ehw106, indexed in Pubmed: 27222591.

14. World Health Organization (WHO) Consultation. Definition and diagnosis of diabetes and intermediate hyperglycaemia. 2006 . http://www.who.int/diabetes/publications/Definition\%20and\%20 diagnosis\%20of\%20diabetes_new.pdf.

15. World Health Organization (WHO), Abbreviated report of aWHO consultation. Use of glycated hemoglobin (HbA1c) in the diagnosis if diabetes mellitus. 2011. http://www.who.int/diabetes/ publications/diagnosis_diabetes2011/en/.

16. Touboul PJ, Hennerici MG, Meairs S, et al. Mannheim Carotid Intima-Media Thickness and Plaque Consensus (2004-2006-2011). Cerebrovasc Dis. 2012; 34(4): 290-296, doi: 10.1159/000343145.

17. Iacobellis G, Assael F, Ribaudo MC, et al. Epicardial fat from echocardiography: a new method for visceral adipose tissue prediction. Obes Res. 2003; 11(2): 304-310, doi: 10.1038/ oby.2003.45, indexed in Pubmed: 12582228.

18. Haberka M, Sosnowski M, Zuziak-Pruska J, et al. Extra-media thickness and epicardial fat: comparison of a novel carotid artery ultrasound index and a well-established cardiac magnetic resonance fat quantification method. Nutr Metab Cardiovasc Dis. 2015; 25(8): 763-770, doi: 10.1016/j.numecd.2015.04.011, indexed in Pubmed: 26033393.

19. Eşref A, Mehmet A, Sefer V, et al. Echocardiographic epicardial fat thickness and neutrophil to lymphocyte ratio are novel inflammatory predictors of cerebral ischemic stroke. J Stroke Cerebrovasc Dis. 2014; 23(9): 2328-2334, doi: 10.1016/j.jstrokecerebrovasdis.2014.04.028, indexed in Pubmed: 25200242.

20. Altun I, Unal Y, Basaran O, et al. Increased epicardial fat thickness correlates with aortic stiffness and n-terminal pro-brain natriuretic peptide levels in acute ischemic stroke patients. Tex Heart Inst J. 2016; 43(3): 220-226, doi: 10.14503/thij-15-5428.

21. Cosansu K, Yilmaz S. Is epicardial fat thickness associated with acute ischemic stroke in patients with atrial fibrillation? J Stroke Cerebrovasc Dis. 2020; 29(7): 104900, doi: 10.1016/j.jstrokecerebrovasdis.2020.104900, indexed in Pubmed: 32402718.

22. Gürdal A, Keskin K, Orken DN, et al. Evaluation of epicardial fat thickness in young patients with embolic stroke of undetermined source. Neurologist. 2018; 23(4): 113-117, doi: 10.1097/ NRL.0000000000000182, indexed in Pubmed: 29953033.

23. Cho KI, Kim BJ, Cho SH, et al. Epicardial fat thickness and free fatty acid level are predictors of acute ischemic stroke with atrial fibrillation. J Cardiovasc Imaging. 2018; 26(2): 65-74, doi: 10.4250/jcvi.2018.26.e1, indexed in Pubmed: 29971268.

24. Acet H, Ertaş F, Akıl MA, et al. New inflammatory predictors for non-valvular atrial fibrillation: echocardiographic epicardial fat thickness and neutrophil to lymphocyte ratio. Int J Cardiovasc
Imaging. 2014; 30(1): 81-89, doi: 10.1007/s10554-013-0317-4, indexed in Pubmed: 24162180.

25. Soeki T, Sata M. Role of epicardial adipose tissue in atrial fibrillation. Circ J. 2012; 76(12): 2738-2739, doi: 10.1253/circj.cj-121283, indexed in Pubmed: 23095722.

26. Pengfei S, Lishun L, Chengzhang L, et al. Carotid intima media thickness and the risk of first in patients with hypertension. Stroke. 2020; 51(2): 379-386.

27. Kawai T, Ohishi M, Takeya Y, et al. Carotid plaque score and intima media thickness as predictors of stroke and mortality in hypertensive patients. Hypertens Res. 2013; 36(10): 902-909, doi: 10.1038/hr.2013.61, indexed in Pubmed: 23823172.

28. Verhagen SN, Visseren FLJ. Perivascular adipose tissue as a cause of atherosclerosis. Atherosclerosis. 2011; 214(1): 3-10, doi: 10.1016/j.atherosclerosis.2010.05.034, indexed in Pubmed: 20646709.

29. Baker AR, Silva NF, Quinn DW, et al. Human epicardial adipose tissue expresses a pathogenic profile of adipocytokines in patients with cardiovascular disease. Cardiovasc Diabetol. 2006; 5: 1, doi: 10.1186/1475-2840-5-1, indexed in Pubmed: 16412224.

30. Bertaso AG, Bertol D, Duncan BB, et al. Epicardial fat: definition, measurements and systematic review of main outcomes. Arq Bras Cardiol. 2013; 101(1): e18-e28, doi: 10.5935/abc.20130138, indexed in Pubmed: 23917514.

31. Iacobellis G, Willens HJ. Echocardiographic epicardial fat: a review of research and clinical applications. J Am Soc Echocardiogr. 2009; 22(12): 1311-1417, doi: 10.1016/j.echo.2009.10.013, indexed in Pubmed: 19944955.

32. Meijer RI, Serne EH, Smulders YM, et al. Perivascular adipose tissue and its role in type 2 diabetes and cardiovascular disease. Curr Diab Rep. 2011; 11(3): 211-217, doi: 10.1007/s11892-0110186-y, indexed in Pubmed: 21461998.

33. Lee HY, Després JP, Koh KK. Perivascular adipose tissue in the pathogenesis of cardiovascular disease. Atherosclerosis. 2013; 230(2): 177-184, doi: 10.1016/j.atherosclerosis.2013.07.037, indexed in Pubmed: 24075741.

34. Berg G, Miksztowicz V, Morales C, et al. Epicardial adipose tissue in cardiovascular disease. Adv Exp Med Biol. 2019; 1127: 131-143, doi: 10.1007/978-3-030-11488-6_9, indexed in Pubmed: 31140176 .

35. Wong CX, Sun MT, Odutayo A, et al. Associations of epicardial, abdominal, and overall adiposity with atrial fibrillation. Circ Arrhythm Electrophysiol. 2016; 9(12): e004378, doi: 10.1161/CIRCEP.116.004378, indexed in Pubmed: 27923804.

36. Le Jemtel TH, Samson R, Ayinapudi K, et al. Epicardial adipose tissue and cardiovascular disease. Curr Hypertens Rep. 2019; 21(5): 36, doi: 10.1007/s11906-019-0939-6, indexed in Pubmed: 30953236.

37. Lin YK, Chen YC, Chen JH, et al. Adipocytes modulate the electrophysiology of atrial myocytes: implications in obesityinduced atrial fibrillation. Basic Res Cardiol. 2012; 107(5): 293, doi: 10.1007/s00395-012-0293-1, indexed in Pubmed: 22886089.

38. Abed HS, Wittert GA. Obesity and atrial fibrillation. Obes Rev. 2013; 14(11): 929-938, doi: 10.1111/obr.12056, indexed in Pubmed: 23879190. 